\title{
Identification of low-frequency kinetic wave modes in the Earth's ion foreshock
}

\author{
X. Blanco-Cano ${ }^{1}$ and S. J. Schwartz ${ }^{2}$ \\ ${ }^{1}$ Instituto de Geofísica, UNAM, Coyoacán, D.F., CP 04510, México \\ 2 Astronomy Unit, Queen Mary and Westfield College, Mile End Rd., London E1 4NS, United Kingdom
}

Received: 10 April 1996 / Revised: 27 September 1996 / Accepted: 8 October 1995

\begin{abstract}
In this work we use ion and magnetic field data from the AMPTE-UKS mission to study the characteristics of low frequency $\left(\omega_{r} \ll \Omega_{p}\right)$ waves observed upstream of the Earth's bow shock. We test the application of various plasma-field correlations and magnetic ratios derived from linear Vlasov theory to identify the modes in this region. We evaluate (for a parameter space consistent with the ion foreshock) the Alfvén ratio, the parallel compressibility, the crosshelicity, the noncoplanar ratio, the magnetic compression and the polarization for the two kinetic instabilities that can be generated in the foreshock by the interaction of hot diffuse ions with the solar wind: the left-hand resonant and the right-hand resonant ion beam instabilities. Comparison of these quantities with the observed plasma-field correlations and various magnetic properties of the waves observed during 10 intervals on 30 October 1984, where the waves are associated with diffuse ions, allows us to identify regions with Alfvénic waves and regions where the predominant mode is the right-hand resonant instability. In all the cases the waves are transverse, propagating at angles $\leq 33^{\circ}$ and are elliptically polarized. Our results suggest that while the observed Alfvén waves are generated locally by hot diffuse ions, the right-handed waves may result from the superposition of waves generated by two different types of beam distribution (i.e. cold beam and diffuse ions). Even when there was good agreement between the values of observed transport ratios and the values given by the theory, some discrepancies were found. This shows that the observed waves are different from the theoretical modes and that mode identification based only on polarization quantities does not give a complete picture of the waves' characteristics and can lead to mode identification of waves whose polarization may agree with theoretical predictions even when other properties can diverge from those of the theoretical modes.
\end{abstract}

Correspondence to: X. Blanco-Cano

xbc@tonatiuh.igeofcu. unam.mx

\section{Introduction}

The terrestrial ion foreshock is permated by a variety of particle distributions and wave modes (Greenstadt, 1985; Thomsen, 1985; Hoppe and Russell, 1983; Winske et al., 1990). The study and identification of these distributions and magnetic signatures gives information about their interrelation and evolution (Fuselier, 1995; Killen et al., 1995) and is central to understand processes such as the thermalization of the solar wind (Greenstadt, 1985; Thomsen et al., 1990; Schwartz and Burgess, 1991; Dubouloz and Scholer, 1995), shock reformation (Burgess, 1989) and Fermi acceleration (Ellison, 1985; Giacalone et al., 1993)

Numerous works have studied the plasma waves observed in the foreshock (Greenstadt et al., 1968; Fairfield 1969; Hoppe et al., 1981; Hoppe and Russell, 1983; Russell et al., 1987; Le, 1991; see special issue of J. Geophys. Res, vol 86, (A6) 1981). However, most of them have relied only on the magnetic field fluctuations and have based their mode identification on MHD dispersion relations. These works have shown that the low frequency $\left(10^{-2} \mathrm{~Hz}\right)$, transverse electromagnetic waves associated with diffuse ions have large amplitudes ( $\sim 5 \mathrm{nT}$ peak to peak) and often steepen and show the formation of shocklets at their upstream edge. These fluctuations have been observed with left-hand and right-hand polarizations, that due to Doppler shift correspond respectively to right-hand and left-hand polarizations in the plasma frame. Comparison to cold plasma dispersion relations identifies these as magnetosonic and Alfvén waves (Hoppe and Russell, 1983). Since waves in the foreshock also have plasma signatures a complete description of the waves requires considering the fluctuation of the proton velocity and density in addition to the magnetic fluctuations. Moreover, because the plasma in the ion foreshock is hot, with a plasma beta $\beta \geq 1$, and because kinetic effects such as the generation of ion instabilities by back- 
streaming ions play an important role in this region, the use of a kinetic model to derive plasma wave identifiers is imperative.

There are other problems related to the identification of space plasma waves based on the waves' frequency $\omega_{r}$ and propagation vector $\mathbf{k}$. Due to the super-sonic solar wind flow the waves suffer a large Doppler shift. This often masks the underlying plasma frequency and complicates the identification of particular modes. In addition, single spacecraft observations can not determine uniquely the phase velocity of the waves, nor the direction of $\mathbf{k}$ with the use of only magnetic field data. As we show later, the use of the cross-helicity (involving fluctuations of the proton velocity) can remove the ambiguity given by the minimum variance analysis in the direction of $\mathbf{k}$. On the other hand, when multiple spacecraft observations are available, it is not always possible to determine the direction of $\mathbf{k}$. The spacecraft need to be located within one coherence length in order to be studying the same wave. Hence, it is of great importance to establish new schemes in which wave mode identification does not depend uniquely on $\omega_{r}, \mathbf{k}$, and magnetic polarization, and in which other properties of the waves, such as plasma-field correlations, are used to identify different modes in a system.

The objective of this work is to characterize the wave properties more completely by systematically considering fluctuations in the proton density and velocity together with the magnetic field measurements. We use AMPTE-UKS ion and magnetic field data to evaluate several transport ratios (Alfvén ratio, cross-helicity, parallel compressibility, magnetic compression and magnetic transverse ratio $R_{n c}$ ) of upstream low frequency $\left(\omega_{r} \ll \Omega_{p}\right)$ waves and compare them with the values obtained from linear kinetic theory for the lefthand resonant and the right-hand resonant instabilities. These instabilities can be generated in the foreshock by the interaction of a hot beam such as the diffuse ions with the solar wind. For both instabilities the ions in the beam are cyclotron resonant with the wave providing the free energy necessary for the wave to grow. The first of these modes evolves out of the Alfvén wave, while the second evolves from the magnetosonic branch. When these two modes propagate at small angles to the magnetic field, the values of the transport ratios can be very similar for both modes (Gary and Winske, 1992), so we also evaluate the sense of polarization of the waves. There are inherent limitations in comparing the results of a single linear mode theory with observations of a nonlinear mixed mode spectrum of waves. Accordingly, we investigate several examples in some detail and consider several transport ratios and other wave characteristics.

To our knowledge, the use of plasma field correlations derived from kinetic theory to identify low frequency waves in the terrestrial foreshock has not been applied systematically in the past. Orlowski et al. (1994b) studied waves upstream of Venus bow shock. Their identification was based on the polarization and magnetic compression of the waves. They compared the properties of different modes derived by Hall-MHD theory with the ones given by the kinetic description (see also Krauss-Varban et al., 1994) and found that while the fast kinetic magnetosonic mode was in excellent agreement with the observed waves, there was not a unique fluid mode that described the observed polarizations, and two Hall-MHD modes were needed to account for the observed wave properties. Evaluating the polarization and general ellipticity from kinetic theory, Orlowski et al. (1994a) showed the existence of the left-hand resonant instability (in their terminology "Alfvénic beam mode"), as well as of the resonant and nonresonant fast magnetosonic modes in the upstream region of Saturn. Nonetheless, in these two works only magnetic correlations were used without considering the fluctuations of the plasma properties, which are essential to characterize a mode in a high $\beta$ regime where the plasma dominates the field dynamics.

Mirror, fast magnetosonic and Alfvén waves have been identified in the terrestial magnetosheath applying the values of transport ratios derived from MHD theory (Lacombe et al., 1990; Song et al. 1994). Lacombe et al. (1992) found significant differences between the properties of the magnetosheath modes derived from linear Vlasov and Hall-MHD theories, stressing the importance of describing low frequency waves using kinetic theory (Krauss-Varban et al., 1994). The mirror mode was identified in the magnetosheath by Denton et al. (1995), who based their identification on the evaluation of a mode deviation, that measures the "distance" between the theoretical kinetic transport ratios of a particular mode and the observed transport ratios. They use this parameter to determine the mode which best describes the observed fluctuations and measure whether or not the resulting identification is unique. An overview of the methods of mode identification made to date is given in Schwartz et al. (1996).

The purpose of this manuscript is to apply several transport ratios and polarization properties evaluated from linear kinetic theory in order to identify wave modes from AMPTE-UKS ion and magnetic field data taken in the ion foreshock in regions where diffuse distributions were present.

The structure of the study is as follows. The next section describes the parameter space used to derive the theoretical values of the wave identifiers used in this study (the appendix defines the various ratios and polarization used in the analysis). We then describe the instrumental characteristics onboard AMPTE-UKS and explain how the observed transport ratios were calculated. We present a study case detailing the methodology followed for wave mode identification. Subsequent sections summarize the waves' characteristics observed in all analyzed intervals and discuss our results.

\section{Theoretical transport ratios values}

We use the program WHAMP (Rönmark, 1982) to solve numerically the full electromagnetic dispersion relation from linear Vlasov theory (Stix, 1992) and evaluate the polarization, the parallel compressibility, 
the Alfvén ratio, the cross-helicity, and two magnetic ratios $C_{B}$ and $R_{n c}$ (see definitions in appendix) of the lefthand and the right-hand resonant ion beam instabilities for a set of hot beam ion distributions that resemble the characteristics of the diffuse ions observed in the foreshock. When cold reflected ions interact with the solar wind, the right-hand resonant and the right-hand nonresonant instabilities can also be generated. Because in all the data set used for this study the waves are associated with diffuse ions, we base our mode identification on the comparison of the observed transport ratios and polarization with the properties of the two hot beam kinetic instabilities. We consider a three-species plasma immersed in a magnetic field $\mathbf{B}_{\mathbf{0}}=B_{o} \hat{z}$. The plasma components are one electron and two ion populations, with Maxwellian zeroth order distributions (subscripts $p, e$ and $b$ refer to the proton core, the electrons and the ion beam). The two ion populations are drifting with respect to one another with a velocity in the $\mathbf{z}$ direction, $\mathbf{v}_{o}=\mathbf{v}_{o b}-\mathbf{v}_{o p}$, with $\mathbf{v}_{o j}$ the velocity of the $\mathrm{j}$-th species in the electron frame of reference. A complete derivation of the dispersion relations of the two hot beam instabilities is given in Gary (1985, 1993).

The application of linear kinetic theory requires that perturbations in the background fields are small. The waves in the foreshock have amplitudes $\delta B / B_{o} \geq 0.50$. Therefore, the use of linear theory to describe the waves in this region can give us some insight about the properties of the observed modes, but does not describe completely the system and we might expect some discrepancies in the wave characteristics derived from Vlasov theory when compared with observations.

Technical reasons prevented us from evaluating the moments of the diffuse ion distributions associated with all the observed waves. Therefore we evaluate theoretical transport ratios for a set of distributions (see table 1) that covers variations in the diffuse ion properties as reported in the literature. Diffuse ions have been observed with nearly isotropic distributions with typical temperatures of several times $10^{7} \mathrm{~K}$, which at times can be as high as $10^{8} \mathrm{~K}$ (Paschmann et al., 1981), an upstream flow of $0.8-1.2 \quad v_{s w}$ and a highly variable density $0.01-0.8 \mathrm{~cm}^{-3}$ (Tsurutani and Rodriguez, 1981). Case 1 in Table 1 shows a beam with characteristics corresponding to the "typical observed" values for the diffuse ions in the foreshock (Thomsen, 1985). Other cases correspond to variations in the beam density (cases 2 and 3), the beam temperature (case 4) and both the beam density and the temperature (case 5). All these distributions resemble the variations observed at times in the properties of the diffuse ions. Our mode identification is done by comparing the observed wave properties with the values obtained for the wave identifiers at a particular wave number considering these five plasma configurations. We use $k c / \omega_{p}=0.10$ for the right-hand resonant instability and $k c / \omega_{p}=0.12$ for the left-handed mode. At these wave numbers, $\omega / \Omega_{i} \sim 0.10$, similar to the frequency values derived in the plasma rest frame by Hoppe and Russell (1983) for the observed ULF waves. With the exception of cases 2
Table 1. Plasma input distributions

\begin{tabular}{ccclc}
\hline & & Protons & Electrons & Beam \\
\hline 1 & $n_{j} / n_{e}$ & 0.99 & 1.0 & 0.01 \\
& $T_{j} / T_{p}$ & 1.0 & 1.0 & 100 \\
& $v_{o j} / v_{\mathrm{a}}$ & -0.02 & 0.0 & 1.98 \\
2 & $n_{j} / n_{e}$ & 0.95 & 1.0 & 0.05 \\
3 & $n_{j} / n_{e}$ & 0.90 & 1.0 & 0.10 \\
4 & $T_{j} / T_{p}$ & 1.0 & 1.0 & 495 \\
5 & $n_{j} / n_{e}$ & 0.95 & 1.0 & 0.05 \\
& $T_{j} / T_{p}$ & 1.0 & 1.0 & 1000 \\
\hline
\end{tabular}

In all cases $v_{A} / c=10^{-4}$ and $m_{p} / m_{e}=1836.1$. Case 1 corresponds to the "fundamental" hot beam distribution, the remaining cases give only the values of the parameters that change, while the rest remain as in case 1 . The ratio $T_{b} / T_{p}$ is given for $\beta_{p}=1 . T_{b}$ is constant but $T_{p}$ varies as $\beta_{p}$ does

and 3 , the growth rate for both instabilities is maximum at wave numbers close to the chosen $k c / \omega_{p}$. The increment in the beam's density shifts the wave number at which the growth is maximum to larger values. The mode properties remain qualitatively the same for a reasonable range of wave numbers in the vicinity of the chosen $k c / \omega_{p}$. Various authors have described the effects that these or similar variations in the ion distributions have on the hot beam instabilities (Gary, 1985, 1993; Gary and Winske, 1992; Blanco-Cano, 1995).

Table 2 contains the values of the transport ratios for the two hot beam instabilities. The range of variation given for each identifier summarizes the variations that the different beam distributions produce in each quantity. Bold characters highlight the values of the identifiers that can be used to distinguish between the two hot beam modes. $P_{B_{o} k}$ and $R_{n c}$ are useful identifiers to distinguish between the right-handed and the lefthanded instabilities for all the considered values of $\beta_{p}$ and $\theta_{k B_{o}}$. Note, that the polarization sense is given in the plasma rest frame and this can be reversed when the wave is observed in the spacecraft frame. At $\beta_{p}<0.20$ the parallel compressibility of the left-handed wave is larger than the observed values for the right-handed mode, and $C_{\| p}$ can be useful to separate between the two modes. As $\theta_{k B_{o}}$ increases, the properties of the two modes diverge one from the other, the right-handed wave becomes compressive with larger values of $C_{B}$ than the Alfvénic mode. The values of the Alfvén ratio and the cross-helicity are very similar for the two modes for almost all $\beta_{p}$ and $\theta_{k B_{o}}$. Only at large $\beta_{p}$ and $\theta_{k B_{o}} \geq 30^{\circ}$ are some differences observed and these two identifiers may be used in addition to other identifiers to distinguish the two hot beam instabilities.

With the exception of the polarization, all the wave identifiers used here are independent of reference frame (see Appendix A in Denton et al., 1995). This is very important to avoid problems associated with Doppler shift or normalization to a parameter that is not fixed from one episode to the next, which can lead to ambiguous identification of a given mode. We explain later how the ambiguity in the sense of polarization can be removed using the cross-helicity. Our chosen set of 
Table 2. Transport ratios

\begin{tabular}{|c|c|c|c|c|c|c|c|c|}
\hline & $\beta_{p}$ & $\theta_{k B_{o}}$ & $P_{B_{o} k}$ & $C_{\| p}$ & $r_{A p}$ & $\sigma_{c p}$ & $C_{B}$ & $R_{n c}$ \\
\hline \multirow{5}{*}{$\begin{array}{l}\mathrm{RH} \text { res. inst. } \\
\text { Hot beam } \\
k c / \omega_{p}=0.10\end{array}$} & $\leq 0.20$ & $(0,30)$ & $(1,4)$ & $\sim 1$ & $\sim 1$ & $\sim-1$ & $\leq 0.25$ & $(0.05,0.50)$ \\
\hline & & $(30,50)$ & $(2,9)$ & $\sim 1$ & $\sim 1$ & $(-0.90,-0.60)$ & $(0.20,0.60)$ & $\leq \mathbf{0 . 3 0}$ \\
\hline & $(0.20,0.70)$ & $(0,30)$ & $(1,5)$ & $(0.80,1.60)$ & $(0.90,1.8)$ & $(-0.90,-0.60)$ & $\leq 0.25$ & $(0.05,0.50)$ \\
\hline & & $(30,50)$ & $(2,11)$ & $(1,1.70)$ & $(1,2)$ & $(-0.90,-0.45)$ & $(0.20,0.60)$ & $\leq \mathbf{0 . 3 0}$ \\
\hline & $(0.70,1.20)$ & $\begin{array}{l}(0,30) \\
(30,50)\end{array}$ & $\begin{array}{l}(1,6) \\
(4,15)\end{array}$ & $\begin{array}{l}(0.4,1.65) \\
(0.90,2)\end{array}$ & $\begin{array}{l}(1,3.5) \\
(\mathbf{1}, \mathbf{3 0}, \mathbf{3 . 7 0})\end{array}$ & $\begin{array}{l}(-1,-0.50) \\
(-0.75,-0.20)\end{array}$ & $\begin{array}{l}\leq 0.25 \\
(0.20,0.60)\end{array}$ & $\begin{array}{l}(0.05,0.50) \\
\leq 0.20\end{array}$ \\
\hline \multirow{6}{*}{$\begin{array}{l}\mathrm{L} H \text { res. inst. } \\
\text { Hot beam } \\
k c / \omega_{p}=0.12\end{array}$} & $\leq 0.20$ & $(0,30)$ & $(-1,-0.35)$ & $(1.20,1.90)$ & $(0.70,1.20)$ & $\sim-1$ & $<0.10$ & $(0.50,1)$ \\
\hline & & $(30,50)$ & $(-0.80,-0.10)$ & $(1.30,2.30)$ & $(0.70,1.15)$ & $(-1,-0.88)$ & $<0.14$ & $(0.60,1)$ \\
\hline & $(0.20,0.70)$ & $(0,30)$ & $(-1,-0.40)$ & $(0.50,1.90)$ & $(0.70,1.20)$ & $\sim-1$ & $<0.10$ & $(0.50,1)$ \\
\hline & & $(30,50)$ & $(-0.80,-0.05)$ & $(0.50,3)$ & $(0.75,1.15)$ & $(-1,-0.88)$ & $<0.14$ & $(0.60,1)$ \\
\hline & $(0.70,1.20)$ & $(0,30)$ & $(-1,-0.20)$ & $(0.10,1)$ & $(0.65,1.15)$ & $\sim-1$ & $<0.10$ & $(0.50,1)$ \\
\hline & & $(30,50)$ & $(-0.80,-0.05)$ & $(-0.10,1.15)$ & $(0.80,1.15)$ & $(-1,-0.85)$ & $<0.14$ & $(0.60,1)$ \\
\hline
\end{tabular}

The values for each parameter cover all the range of values obtained for all the beam distributions considered in each case. Bold characters highlight the values that can be useful to separate the different modes. $\beta_{p}$ is the proton core beta; $\theta_{k B_{o}}$ is the angle of propagation with respect to the background magnetic field; $P_{B_{o} k}$ is the polarization as defined in text; $C_{\| p}$ is the parallel compressibility; $r_{A p}$ is the Alfvén ratio; $\sigma_{c p}$ is the cross-helicity; $C_{B}$ is the magnetic compression and $R_{n c}$, the noncoplanar ratio

parameters includes magnetic ratios as well as plasmafield correlations, providing sufficient information about the observed waves' characteristics. However, because there is not a particular set of ratios that can be proven to correspond to only one theoretical mode, comparison of observed transport ratios with theoretical values can show that observations are consistent with a particular mode, but in some instances may not provide a unique way to identify the observed mode.

\section{AMPTE-UKS data analysis}

The AMPTE-UKS ion instrument (Coates et al., 1985) operated a solar wind mode in which high resolution in angle and energy measurements of an anti-sunward sector accumulated over $0.625 \mathrm{~s}$ were made. The solar wind energy (SWE) distribution was an integration over a $45^{\circ}$ by $45^{\circ}$ region giving a 30 point energy spectrum $(590 \mathrm{eV}-3790 \mathrm{eV})$. The solar wind angular (SWA) distribution consists of five broad energy bands covering the same energy range for each 4 polar $\times 8$ azimuthal angles $\left(11.25^{\circ} \times 5.62^{\circ}\right.$ each angular sector). The instrument returned one SWE and one SWA distribution every $10 \mathrm{~s}$. These data are unique in providing highresolution phase space distributions of the solar wind.

In solar wind mode, the UKS ion instrument's energy sweeps occasionally lost synchronization with the DPU's (data processing unit) accumulation pattern. We developed an algorithm to identify such intervals and remove them from subsequent analysis (BlancoCano, 1995). The bulk speed and the temperature of the solar wind protons were found by fitting the proton distributions to Maxwellians. The velocity direction was found from the SWA distribution, using the energy bin corresponding to the proton peak and taking the moment for the azimuthal and polar angles. The density was found by integrating the SWE proton peak, defined by the points used for fitting their peak. This may underestimate $n$ if the full peak is not contained within the SWE energy range.

We have examined the AMPTE-UKS FTR ion data, in which 3-D ion distributions were given each $5 \mathrm{~s}$ with energies in the range $10 \mathrm{eV}-20 \mathrm{keV}$ (Coates et al., 1985) for evidence of suprathermal ions for each of our study cases and find that in all of them, suprathermal ions with diffuse characteristics are present. Panel a in Fig. 1 shows two minute averages of the solar wind peak and of the suprathermal ion distributions for three intervals. The middle panel illustrates the narrow azimuthal distribution of the solar wind ions and Fig. 1c shows the angular location of the suprathermal particles shown in panel a. The values given for $\phi$, the azimuthal angle, indicate flow direction. $\phi=180^{\circ}$ corresponds to ions travelling antisunward, while ions moving sunwards are observed with $\phi=0^{\circ}$. A solid line is used for the distributions observed during interval 11:14:45-11:16:45 on 30 October 1984, where the number of energetic ions is largest and the angular distribution of this population indicates that their distribution is isotropic as corresponds to diffuse ions. For comparison, the distributions plotted with a dashed line correspond to an interval where a reflected ion beam is present, as indicated by the anisotropic angular distribution shown in panel c, while the interval 12:24:00-12:26:00, provides an example of quieter solar wind conditions, with a larger solar wind speed and a smaller number of counts at suprathermal energies.

The magnetic field measurements are from the UKS triaxial flux gate magnetometer (Southwood et al., 1985). We use high resolution (8 vectors/s) magnetic data when available and low resolution spin averaged data (one vector is given each $5 \mathrm{~s}$ ) for the other cases.

\section{Observed transport ratios}

The SWE ion data were obtained with a cycle time $(\sim 10 s)$ of the order of the wave period observed in 
previous studies, thereby precluding the ability to follow the variations within an individual wave cycle. Instead, we have adopted a statistical approach to determine the correlations between various magnetic quantities which make up the transport ratios and the various magnetic quantities employed here. First we identified intervals containing waves of interest. Ambient conditions $\left(\mathbf{B}_{\mathbf{0}}, \mathbf{v}_{p o}\right.$ and $\left.n_{p o}\right)$ were determined by averaging the observed values over each analyzed interval. All the subsequent analysis was done as a function of time, with one ion data point each $\sim 10 \mathrm{~s}$ and 8 magnetic field vectors per second ( 1 vector each $5 \mathrm{~s}$ ) when high-(low)resolution field data is used. We evaluate the transport ratios for each ion measurement available and then take sliding averages over a period $T_{a}=2$ min every $0.5 \mathrm{~min}$. Therefore, every 2 min we have an average value for each identifier, I, evaluated as

$I=\frac{1}{N_{s}} \sum_{i=1}^{N_{s}} I_{i}$

where $I_{i}$ is the value of the identifier for each data point within the sliding interval with $N_{s}$ ion measurements. To calculate quantities that involve plasma and magnetic field data $\left(r_{A p}, \sigma_{c p}, C_{\| p}\right)$ we interpolated the magnetic field corresponding to each ion measurement as follows; when we used high resolution magnetic field data, an average field vector was evaluated, representative of the time during which the ion measurements were taken $(0.625 \mathrm{~s})$. In the case of low resolution magnetic field data the values of the magnetic field corresponding to the ion data points were interpolated using a cubic spline. The values of $C_{B}$ and $R_{n c}$ are evaluated from the reduced magnetic field points.

\section{Results}

On 30 October 1984, the AMPTE-UKS spacecraft was in the ion foreshock for almost three hours, starting at 11:00:00. We have selected 10 intervals that are representative examples of each type of wave to describe their characteristics and illustrate the signatures typically encountered in the foreshock during this day. In this section we present the values of the transport ratios and polarization properties found for the waves in one interval and describe how the identification of the mode was made.

\section{Alfvén mode, left-hand resonant instability}

As an example case to describe how the different wave parameters are used to identify a wave mode, we selected the interval 11:14:45-11:17:20 (event 2 in Tables 3 and 4). This interval corresponds to a location deep in the foreshock, where large amplitude waves are observed in the presence of diffuse ions (see Fig. 1). The plasma and magnetic field data are shown in Fig. 2. The first panels illustrate the proton density, temperature, average beta and the velocity. The last four panels show the high resolution magnetic field, where the waves appear as irregular signatures. A dashed line is used to plot the average values of each quantity (evaluated each $0.5 \mathrm{~min}$ in a sliding window of width $2 \mathrm{~min}$ ). Note, however, that to derive the values of the transport ratios and the polarization characteristics of the waves the average values of the density, the velocity and the magnetic field were taken over all the interval of time presented in each example, rather than the sliding
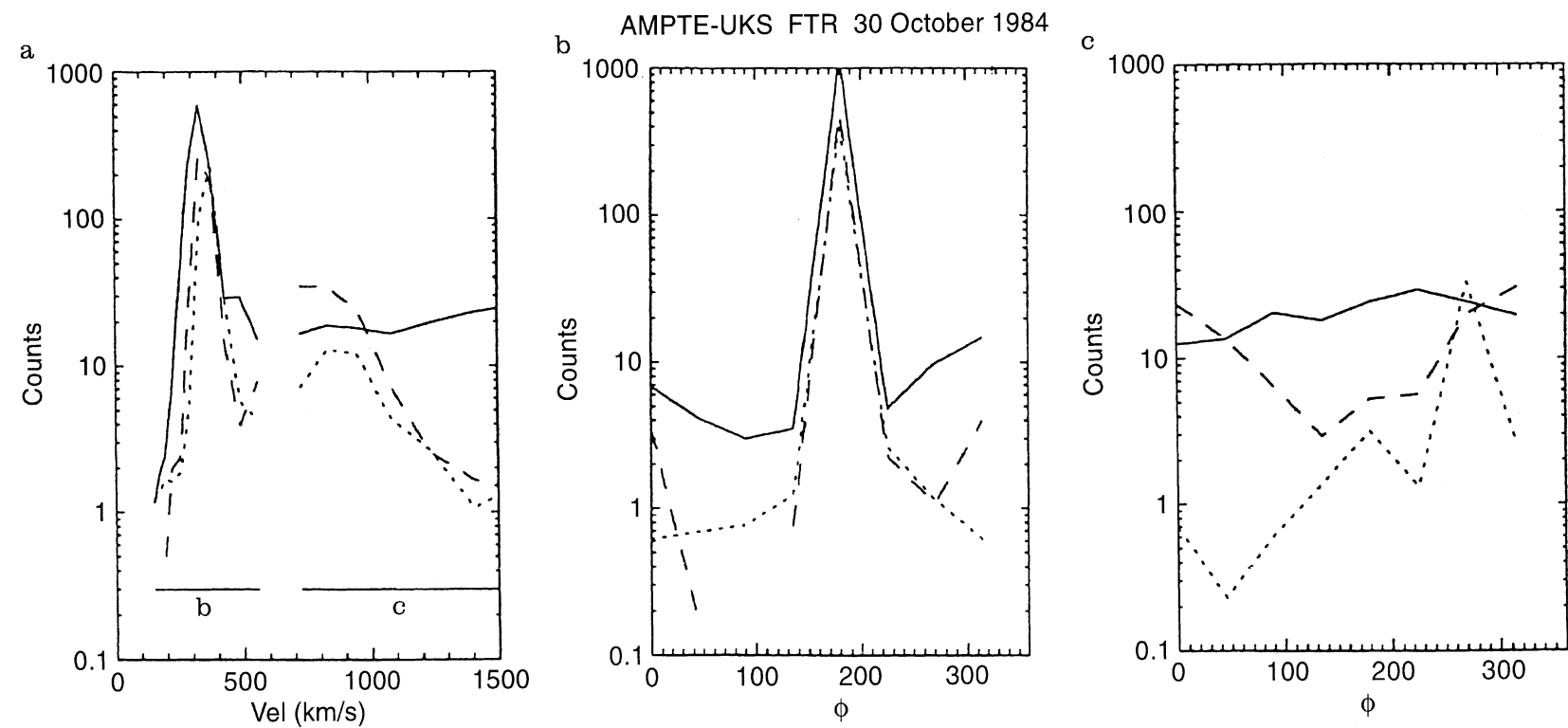

111445-111645 Diffuse

- 133700-133900 Beam

122400-122600 Quiet SW

Fig 1. a shows the velocity distributions of the solar wind ions and of the suprathermal particles. b shows the narrow angular distribution of the solar wind ions (i.e. protons plus alpha particles with measured speeds in the range $148.456-557.193 \mathrm{~km} / \mathrm{s}$ as shown in a. $\mathbf{c}$ shows the angular location of the suprathermal particles (with speeds between $725.916-1832.190 \mathrm{~km} / \mathrm{s}$ ) 


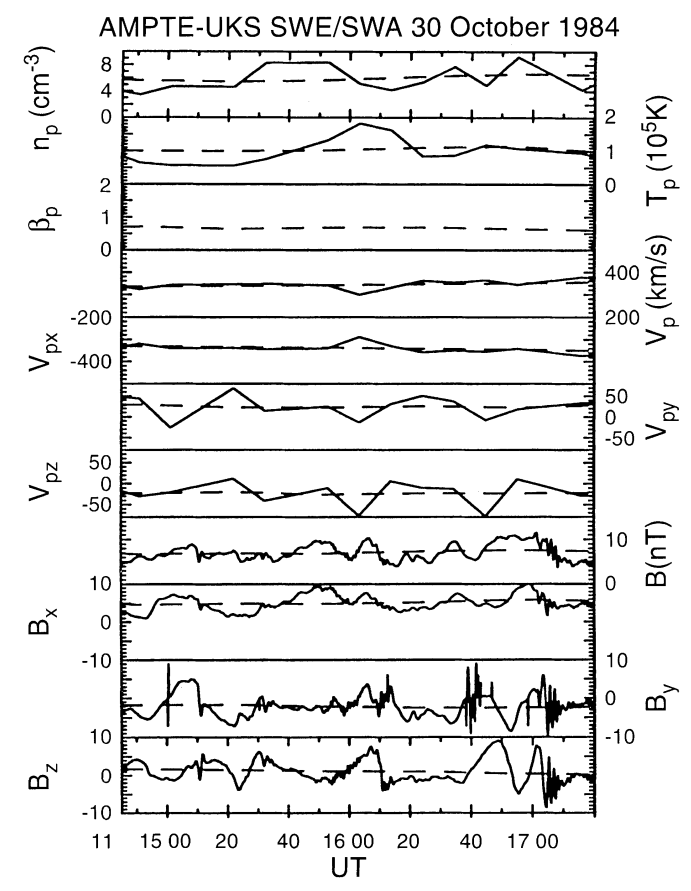

Fig 2. Plasma and magnetic field data (solid line) and average values (dashed line) corresponding to the interval 11:14:45-11:17:20 on 30 October 1984. From top to bottom the panels display $n_{p}$, the proton density; $T_{p}$, the proton temperature; $\beta_{p}$, the proton beta; $v_{p}$, the velocity magnitude; $v_{p x}, v_{p y}$ and $v_{p z}$, the velocity components; $B$ the magnetic field magnitude; $B_{x}, B_{y}$ and $B_{z}$, the magnetic field components

averages. From the density, velocity and magnetic field panels it can be seen that for this case the averages over the sliding window remain fairly constant in all this region. Thus, our evaluation of $n_{p o}, \mathbf{v}_{p o}$ and $\mathbf{B}_{\mathbf{o}}$ is appropriate.

Figure 3 shows 2 min average values (each $0.5 \mathrm{~min}$ ) of the waves' transport ratios and magnetic properties for this interval. The left-hand panel displays the observed plasma-field correlations (Alfvén ratio, cross-helicity, parallel-compressibility) and magnetic ratios (magnetic compression, noncoplanar ratio, magnitude of polarization) that we use for mode identification. The values of these identifiers provide a new piece of information about the characteristics of upstream fluctuations. The right panel shows the group of magnetic properties (definitions given in Appendix) that besides power spectrum analysis has been commonly used in the past for the identification of waves in the foreshock. Additionally, as mentioned already, some authors (Hoppe and Russell, 1983) have evaluated plasma rest frame frequencies and wave vectors and compare their results with $\mathrm{MHD}$ dispersion relations.

We do not evaluate the exact moments of the observed diffuse ions for technical reasons. The characteristics of the diffuse ions present in foreshock regions like the one we study here are well described by a distribution within the set of hot beam distributions given in Table 1, for which we derived the properties of the hot beam modes. During this interval the average value for the proton beta is $\beta_{p}=0.67$ and the waves propagate at angles $\theta_{k B_{o}} \approx 12^{\circ}$ (or $168^{\circ}$ ) to $\mathbf{B}_{\mathbf{0}}$. The $180^{\circ}$ ambiguity in the direction of $\mathbf{k}$ is removed using the sign of the cross-helicity. In this region $\sigma_{c p}=-0.64(<0)$ indicating that the waves propagate at angles $<90^{\circ}$ to the ambient magnetic field. Since $\mathbf{B}_{\mathbf{0}}=(5.457,-2.364$, $-0.984) \mathrm{nT}$ we conclude that the waves are propagating upstream in the plasma rest frame at an angle $\theta_{k B_{o}} \approx 12^{\circ}$. When we compare the observed values of the Alfvén ratio $\left(r_{A p} \approx 1.02\right)$, the cross-helicity $\left(\sigma_{c p} \approx-0.64\right)$, the parallel compressibility $\left(C_{\| p} \approx 0.78\right)$, the magnetic compression $\left(C_{B} \approx 0.15\right)$, the transverse ratio $\left(R_{n c} \approx 0.44\right)$ and the magnitude of the polarization $\left(\left|P_{B_{o} k}\right| \approx 1.05\right)$ with the values derived by the theory for waves with $\theta_{k B_{o}} \leq 30^{\circ}$ (see Table 2) we see that they are in good agreement with the values for both the left-hand resonant instability and the right-hand resonant mode. As mentioned earlier, when these two modes propagate

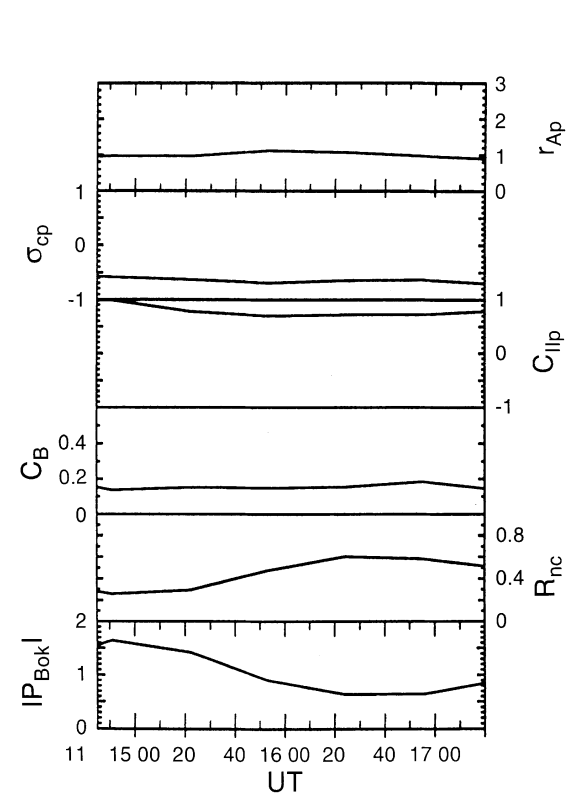



Fig 3. Waves' transport ratios and magnetic properties evaluated for the same period as Fig. 2. In this example high resolution magnetic field was used. The left panel shows the transport ratio values and polarization $P_{B_{o} k}$. The right-hand panel displays the values of $\theta_{k B_{o}}$ (solid line) and $\theta_{i B_{0}}$ (dashed line); $\delta B_{n} ; \epsilon$ (solid line) and $e$ (dashed line); $P_{\text {mean }}$ (solid line) and $s r$ (dashed line); $f_{r} ;$ and $B$ 
at small angles to $\mathbf{B}_{\mathbf{0}}$, their properties are very similar, and it is necessary to use the sense of polarization, or the helicity in addition to the plasma-field correlations (Gary and Winske, 1992) to make a certain identification of the mode. The waves in this interval are righthand polarized in the spacecraft frame $(s r>0)$. Knowing that the waves are propagating upstream in the plasma rest frame (since $\sigma_{c p}<0$ ) and that the hot beam low frequency modes have phase speeds much smaller than the solar wind speed, the sense of polarization in the spacecraft frame will be reversed to that in the plasma rest frame (Hoppe et al., 1981; Hoppe and Russell, 1983). We conclude that the waves in this region are left-hand polarized in the plasma rest frame, and can be identified as an Alfvén/left-hand resonant instability.

The values of the magnetic properties reveal that these observed Alfvén waves are transverse $\left(\theta_{i B_{o}} \approx 85^{\circ}\right)$, have large amplitudes $\left(\delta B_{n}=0.79\right)$ and are elliptically polarized $(\epsilon \sim 1.35)$. The value of $e \approx 0.40$ manifests that the waves are not purely planar in nature. The majority of the waves are right-handed in the spacecraft frame $(s r>0)$, but large rotations occur in the opposite sense $\left(P_{\text {mean }}<0\right)$. When we evaluate $P_{\text {mean }}$ using low resolution magnetic field data, we find $P_{\text {mean }}>0$ throughout the interval. Thus, the left-handed large rotations are associated with high frequency fluctuations superimposed on the low frequency large amplitude waves, which for this interval are right-handed in the spacecraft frame. This is similar to previous results (Tsurutani et al., 1987; Muret and Omidi, 1995) showing the existence of higher frequency waves with a different sense of polarization superimposed on the low frequency fluctuations. The "rotational" frequency $\left(f_{r} \approx 0.130 \mathrm{~Hz}\right)$

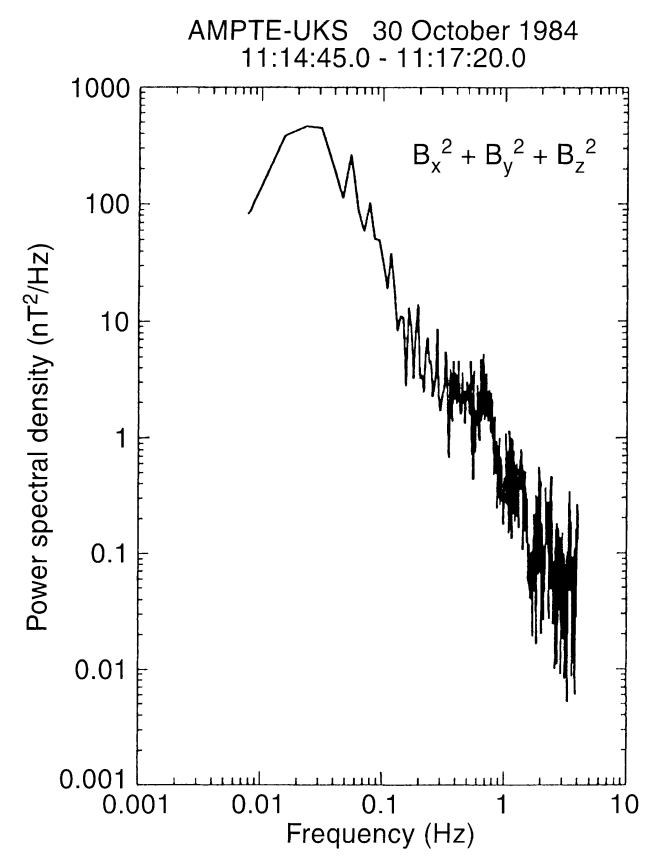

Fig. 4. Power spectrum of magnetic field fluctuations (high resolution). The power spectra density corresponds to the sum of the power in each of the components. The Nyquist frequency is $f_{N y q}=4 \mathrm{~Hz}$

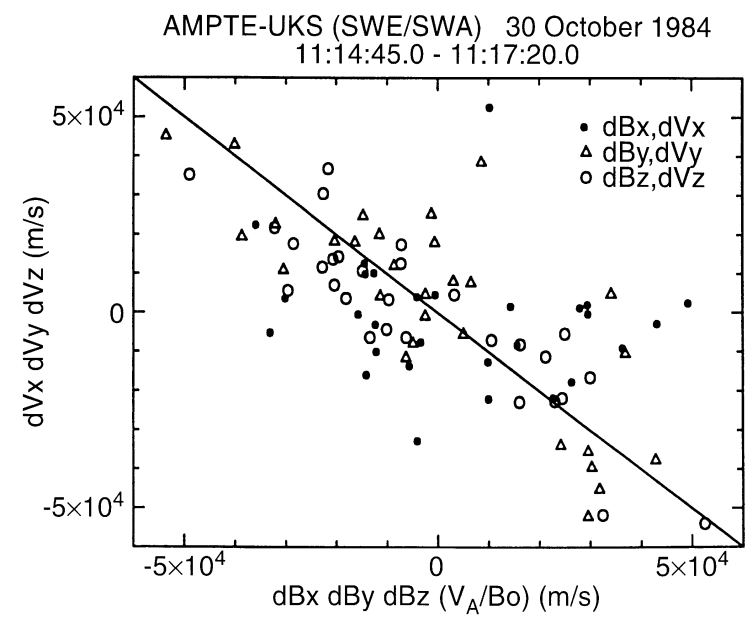

Fig. 5. Correlation between the components of the velocity fluctuations, $\delta \mathbf{v}$ and the corresponding magnetic components $\delta \mathbf{B}$ for the interval 11:14:45-11:17:20. During this interval $r_{A p}=1.02$ and $\theta_{k B_{o}}=12^{\circ}$. The solid line is the expected relation $\delta \mathbf{v}=-\delta \mathbf{B} /$ $\left(v_{A} / \mathbf{B}_{\mathbf{o}}\right)$ for an Alfvén wave propagating parallel to the background field

is higher than the values of the frequencies that correspond to the power spectral density $(\sim 0.01-$ $0.05 \mathrm{~Hz})$ peaks, shown in Fig. 4. This is also a consequence of the small amplitude large rotations in the magnetic field fluctuations.

In Fig. 5 the $\delta \mathbf{v}$ components are plotted versus $\delta \mathbf{B}\left(v_{A} /\left|\mathbf{B}_{\mathbf{o}}\right|\right)$ for the waves observed in this interval. As expected for an Alfvén mode propagating quasiparallel to the unperturbed magnetic field, $\delta \mathbf{v} \sim-\delta \mathbf{B}\left(v_{A} /\left|\mathbf{B}_{\mathbf{o}}\right|\right)$.

\section{Summary of observations}

Within the day of observations from AMPTE-UKS used for this study, we selected ten intervals of data in which the evaluated characteristics remained fairly constant for each interval. In all the studied intervals, diffuse ion distributions are observed. Panels $a$ and $b$ in Fig. 6 show velocity and angular distributions for the suprathermal particles associated to events $1-6$ in Tables 3 and 4, while distributions in panels $\mathrm{c}$ and d correspond to events $7-10$. These distributions show that in all the events the suprathermal particles have relatively flat energy spectra at large energies and broad angular distributions, with $T_{b}>10^{7} \mathrm{~K}$.

Figures 7 and 8 display a comparison between the observed transport ratios and the values derived from linear kinetic theory for the two hot beam instabilities. The theoretical values are given for all the beam distributions in Table 1 for a range in $\beta_{p}$ values that covers the observed solar wind betas. The observed average quantities (Table 3) are plotted with an error bar given by their associated standard deviation.

In Fig. 7 diamonds correspond to the properties of the waves that are right-handed $(s r>0)$ in the spacecraft frame (events $1-6$ in Tables 3 and 4), while solid dots are used for events 7-10 (left-hand polarization in 

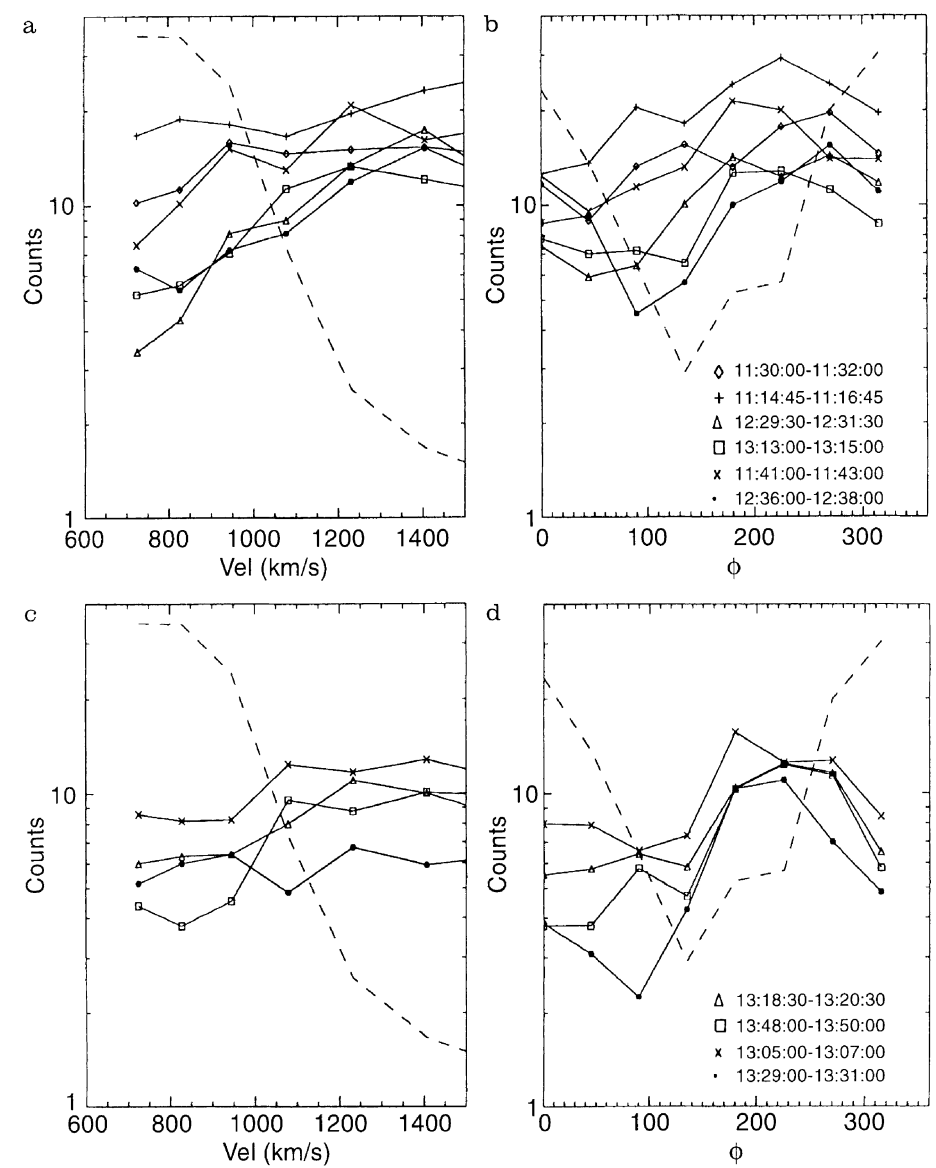

Fig. 6. a and c show $2 \mathrm{~min} /$ averaged velocity distributions (with speeds between $725.916-1832.190 \mathrm{~km} / \mathrm{s}$ ) of the suprathermal particles observed at the times corresponding to events $1-5$ and 6-10 respectively in Table 3 . b and $\mathbf{d}$ show the angular location (azimuthal angle) of the distributions shown in a and $\mathbf{c}$ respectively. In all panels the dashed line corresponds to a two minute averaged distribution of an interval (13:37:00-13:39:00) where an anisotropic reflected ion beam was observed the spacecraft frame, $s r<0)$. In all the events $\sigma_{c p}<0$ and we use this value to remove the $180^{\circ}$ ambiguity in the minimum variance direction. The waves are moving upstream in the plasma rest frame and provided that their phase velocities are smaller than the solar wind velocity, they suffer a reversal in their sense of polarization. Thus, data points indicated with diamonds correspond to waves that are left-handed in the plasma frame, while dotted points correspond to waves with intrinsic right-hand polarization. In Fig. 7, the value of the polarization $P_{B_{0} k}$ is given including the sense of polarization in the plasma frame for the events with intrinsic left-hand polarization and it is possible to see that for these waves the observed values of the magnitude of $P_{B_{0} k}$ are always larger than the values predicted by the theory. For most of our studied cases, the observed parallel compressibilities are within the range of theoretical values derived for the left-hand resonant instability, while the majority of the observed Alfvén ratio values are smaller than the theoretical curves (except events 2 and 8). The observed crosshelicities have smaller magnitudes than the theoretical value $\left|\sigma_{c p}\right| \sim 1$, with the larger discrepancies present for the waves that are right-hand polarized in the plasma rest frame. In all the events, the observed values of the magnetic compression $C_{B}$ are larger than the theoretical curves, while observed $R_{n c}$ values are smaller than the ones predicted by the theory.
Even if some discrepancies exist between the observed values of $P_{B_{o} k}, C_{\| p}, r_{A p}, \sigma_{c p}, C_{B}$ and $R_{n c}$ and the values given by the theory, we believe that the waves in events 1-5 (diamonds), with intrinsic left-hand polarization, can be identified as the left-hand resonant instability. For event 6 (Table 3 ) the cross-helicity magnitude is very small, in disagreement with the theoretical value predicted for the Alfvén instability. Nevertheless, for this event the deviations between the rest of the observed transport ratios and the theoretical values are very similar to the differences found for the other intrinsically left-hand polarized events. So these waves have also some Alfvénic characteristics. From the observed magnetic ratios and $P_{B_{0} k}$ we realize that even in the events identified as Alfvénic, the magnetic field fluctuations presented a significant compressive component (in the plane $\mathbf{k}-\mathbf{B}_{\mathbf{o}}$ ), and are not completely Alfvénic.

In Fig. 8 we compare the observed transport ratios with the theoretical properties of the hot beam righthand resonant mode. Diamonds indicate intrinsically left-hand polarized waves (events 1-6), while solid dots indicate right-handed waves (events 7-10). Observed values of $P_{B_{o} k}$ and $R_{n c}$ are in very good agreement with the theory, for the waves that are right-handed in the plasma rest frame. For most of the events, there are some discrepancies between the observed and theoretical plasma-field correlations values. The observed parallel 

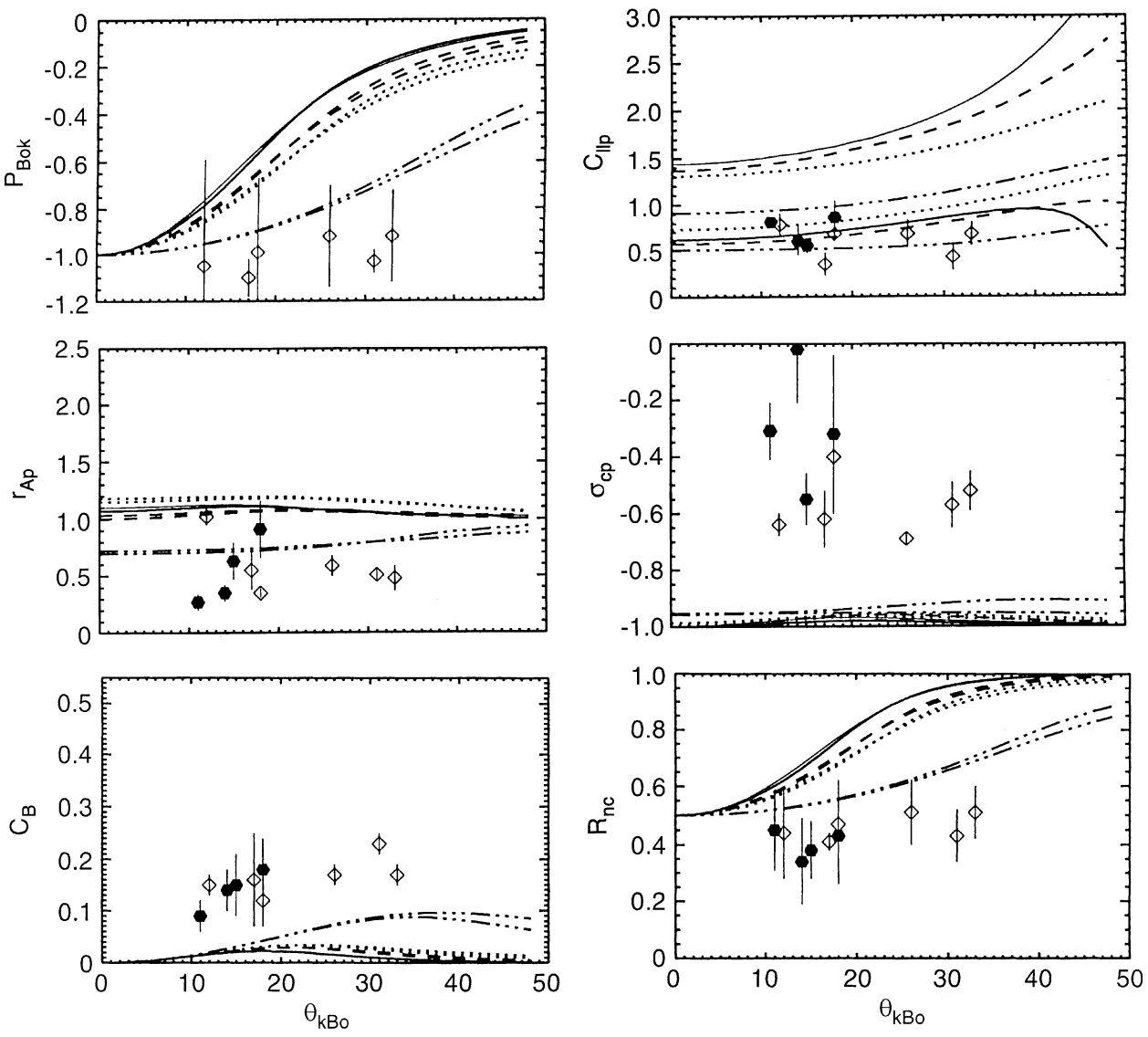

LH-Resonant instability

$\beta_{p}=0.40-0.80 \quad$ Case $1 \quad \cdots$ Dense, case $2 \quad-$ Hot, case $4 \quad-\cdot$ Dense, hot, case 5
Fig. 7. Observed transport ratios and polarization comparison with the values derived from linear kinetic theory for the left-hand resonant instability $\left(k c / \omega_{p}=0.12\right)$. Diamonds correspond to events $1-6$ in Table 3 (left-handed waves in the plasma rest frame), while solid dots are used for events 7-10 (right-handed waves in plasma rest frame) compressibilities and Alfvén ratios are in general smaller than the theoretical curves, while the magnitude of the cross-helicity for the intrinsically right-handed waves is smaller than the theoretical values. For the waves observed during event 7 , with an angle of propagation $\theta_{k B_{o}}=15^{\circ}$, the cross-helicity is $\sigma_{c p} \sim-0.55$. For events $8-10,\left|\sigma_{c p}\right|$ is even smaller being in disagreement with the theoretical predictions for modes propagating at small angles to the magnetic field. Such small cross-helicities may not be reliable to remove the $180^{\circ}$ ambiguity in the minimum variance direction. For events 7-10 (with intrinsic right-hand polarization), the magnetic compression $C_{B}$ is larger than the values predicted by the theory indicating that the observed waves are more compressive than the right-hand linear mode. The intrinsically lefthanded waves with larger angles of propagation $\left(\theta_{k B_{o}}=26-31^{\circ}\right)$, have cross-helicities and magnetic compression values that match the theoretical values of the right-hand resonant instability, indicating that these waves identified as Alfvénic are not $100 \%$ Alfvènic.

From the values of the plasma-field correlations and the sense of polarization we identify event 7 as the hot beam right-hand instability (parallel magnetosonic), and believe that waves in events 8-10 may be identified as the hot beam right-hand resonant mode, but the small $\sigma_{c p}$ makes mode identification uncertain as indicated by a question mark in Table 3.
The right-hand resonant mode can also be generated by cold beams such as the reflected beams observed in the foreshock. As mentioned already, because in all the studied intervals diffuse ions are present, we base our mode identification on the theoretical properties of the two hot beam resonant modes. However, when we compare the properties of the waves observed with intrinsic right-hand polarization (events 7-10) with the transport ratios for the cold beam right-hand resonant instability (Fig. 9), we find that with the exception of the Alfvén ratio, which for this instability is $r_{A p} \gg 1$, all the observed transport ratios are close to the values obtained for the cold beam right-hand resonant mode. For this mode $\left|\sigma_{c p}\right|$ has small values at all angles of propagation. Thus, it is possible that some of the observed right-handed waves are not generated locally by the hot beam instability, but in other regions of the foreshock by reflected cold beams and then are convected by the solar wind flow to regions deep in the foreshock, where diffuse ions are present and the generation of the hot beam mode takes place. Therefore, the observed right-handed waves may result from the superposition of right-handed modes generated by two different types of beam distribution. The fact that we never observe large Alfvén ratio values suggests that some of the characteristics of the waves generated by cold beams may change as they propagate and interact 
with the diffuse ions. In particular, the waves can steepen as a result of finding a strong spatial gradient in the density of diffuse ions (Scholer, 1993; Dubouloz and Scholer, 1995) and nonlinear processes may be important in changing the properties of the observed waves.

From Fig. 7 and 8, is clear that the values of the waves' transport ratios observed with intrinsic left-hand and right-hand polarization are comparable, indicating that the properties of the waves are very similar and that in all the events, the observed waves present a compressive component that is in contrast to the incompressive character predicted by linear theory for low frequency modes at small angles of propagation.

The values of the magnetic wave characteristics averaged over each interval together with their associated standard deviation are given in Table 4. The wave frequencies are of the order of $10^{-2} \mathrm{~Hz}$ in all the data set, they propagate at angles $\leq 33^{\circ}$ and have very large amplitudes with $\delta B_{n} \geq 0.5$ in all the events. The value of $e=\lambda_{3} / \lambda_{2}$ is not very small for the majority of the intervals implying that the wave fields are not completely constrained to a plane. In all the events the waves are elliptically polarized. For all the events except event 2, the mean polarization $P_{\text {mean }}$ and $s r$, the polarization given by number have the same sign. In case 2, large rotations associated with small amplitude, high-fre- quency waves occur in the opposite sense given by $s r$ and this changes the sign of $P_{\text {mean }}$.

\section{Discussion and conclusions}

The low-frequency waves observed in the Earth's ion foreshock by AMPTE-UKS have been described in terms of their transport ratios (Alfvén ratio, crosshelicity, parallel compressibility, magnetic compression and magnetic transverse ratio) and polarization properties. Large amplitude waves were observed with righthand and left-hand polarizations. We were able to identify regions where the waves characteristics indicate the presence of the Alfvén/L-H resonant instability as the predominant mode and regions where the waves are magnetosonic (R-H resonant instability).

Our mode identification is based on the comparison between the observed waves' characteristics and the properties described for low frequency ion instabilities in a linear kinetic model. When the magnetosonic mode is propagating at small angles to the magnetic field in a small $\beta_{p}$ plasma it is Alfvén like (e.g. $\delta \mathbf{B}^{2} / \mathbf{B}_{\mathbf{0}}{ }^{2} \gg$ $\left.|\delta n|^{2} / n^{2}\right)$. Under such conditions the right-hand and left-hand resonant instabilities have similar characteristics at small angles of propagation and mode
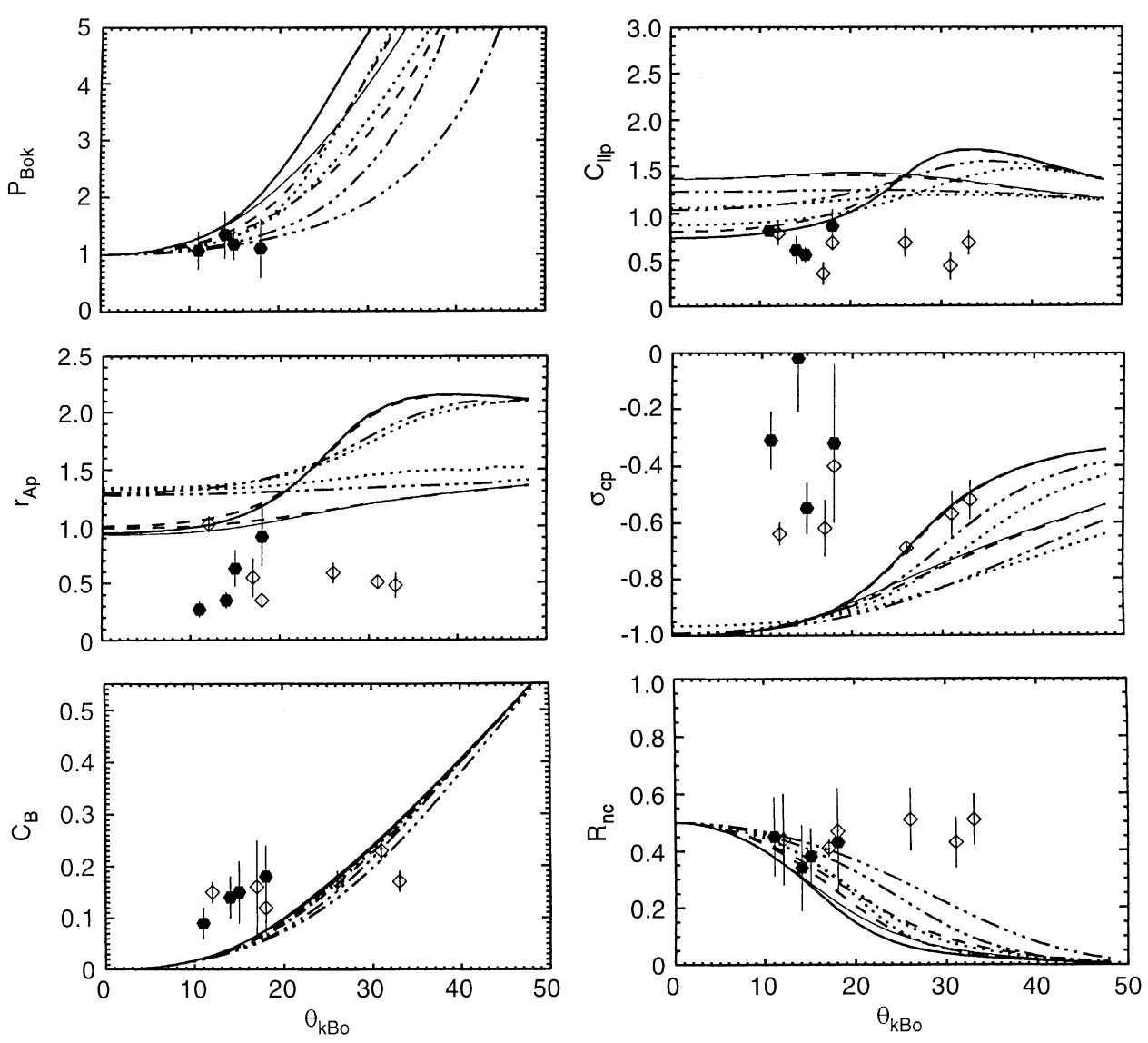

RH-Resonant instability

$\beta_{p}=0.35-0.80 \quad$ Case $1 \quad \cdots$ Dense, case $3 \quad-$ Hot, case $4 \quad-\cdot$ Dense, hot, case 5
Fig. 8. Observed transport ratios and polarization comparison with the values derived from linear kinetic theory for the right-hand resonant instability $\left(k c / \omega_{p}=0.10\right)$. Solid dots correspond to events $7-10$ in Table 3 (right-handed waves in plasma rest frame), while diamonds are used for events 1-6 (left-handed waves in the plasma rest frame) 
identification can not be based only on plasma-field correlations $\left(r_{A p}, C_{\| p}\right.$, and $\left.\sigma_{c p}\right)$, but requires the evaluation of either the magnetic polarization, the helicity (Gary and Winske, 1992) or the magnetic ratio $R_{n c}$. Lefthand and right-hand polarized waves in the foreshock tend to have a compressive component in the plane $\left(\mathbf{B}_{\mathbf{0}}, \mathbf{k}\right)$, and the values of $R_{n c}$ are similar for the observed left-handed and right-handed waves, so that even for waves at angles of propagation $\theta_{k B_{o}} \geq 25^{\circ}$ where $R_{n c}$ could be used to separate the two modes, the discrepancies between observed and theoretical values eliminate such a possibility. Therefore, it is imperative to consider the plasma rest frame polarization or the helicity beside the plasma-field correlations to obtain a valid mode identification.

The cross-helicity is useful to remove the $180^{\circ}$ ambiguity in the direction of $\mathbf{k}$. However there are four events $(6,8,9,10$ in Table 3$)$ in which all the wave properties correspond to one mode except the crosshelicity, which takes small values compared with the ones given by the theory (see Fig. 7 and 8 ). Small values for the cross-helicity can be observed when a mixture of waves propagating in opposite directions is present (Gary, 1986; LaBelle et al., 1994). On the other hand, the observed Alfvén ratio values were smaller than the predicted values for most of the events. Matthaeus and Goldstein (1982) found that for waves in the solar wind, the values of $\sigma_{c p}$ and $r_{A p}$ were sensitive to the assumption of incompressibility made by evaluating an average density over each studied interval. Considering instantaneous density values in the normalization they obtained larger values for the cross-helicity and the Alfvén ratio tended toward unity.
In all the presented intervals, the observed magnetic compression $C_{B}$ is larger than the values predicted by the theory, while $R_{n c}$ is smaller, so that the waves are more compressive than the theoretical modes which are nominally incompressive at small $\theta_{k B_{o}}$.

Even if, on general terms, we found reasonable agreement between the values of the transport ratios given by the theory and the observed values, in all the events there were some discrepancies. This may simply be due to several factors that are different between the assumptions made by the theory and the system that is being studied. In particular, linear theory requires small amplitudes whereas the events studied here displayed normalized amplitudes $\sim 0.5-1.20$ so that nonlinear processes may be important. Linear theory is also based on plane waves, but the minimum variance analysis reveals only a marginal determination of $\mathbf{k}$ in many instances. The theoretical transport ratios are calculated from an individual wave, whereas in reality what is observed in the foreshock is the superposition of different modes with different frequencies. This implies that any mode identification is describing the predominant mode existent in each region. On the other hand, we evaluated the theoretical transport ratios by considering a hot isotropic beam while in reality the observed suprathermal distributions are not completely isotropic. Besides, absolute errors in the proton density (e.g. the under-estimation due to the solar wind coverage) can impact directly the value of $\beta_{p}$ and the plasmafield correlations.

The differences found between the observed and theoretical transport ratios values also show that the observed modes are distinct from the theoretical ones
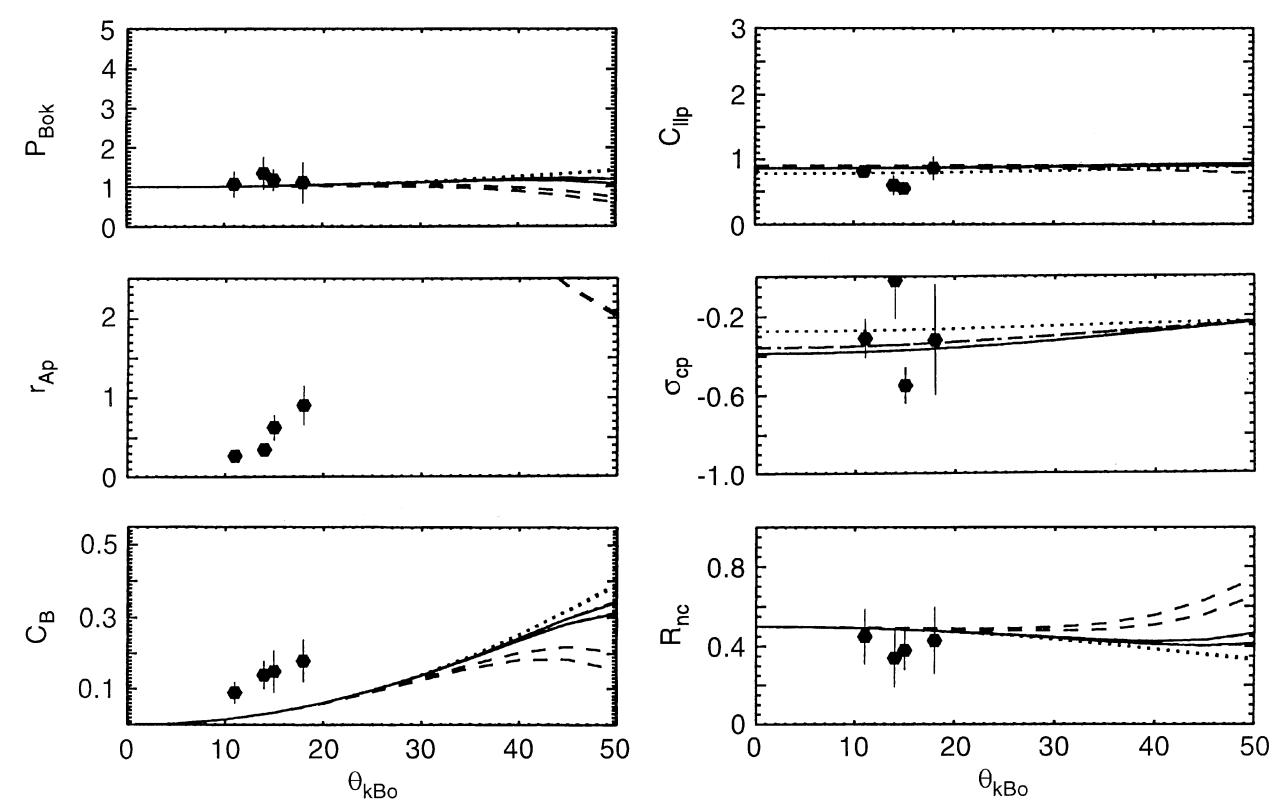

$\mathrm{RH}-$ Resonant instability

$$
\begin{array}{ll}
\mathrm{n}_{\mathrm{b}}=0.01 \mathrm{n}_{\mathrm{e}}, \mathrm{T}_{\mathrm{p}}=\mathrm{T}_{\mathrm{e}} \\
\mathrm{T}_{\mathrm{b}}=10 \mathrm{~T}_{\mathrm{p}} \quad\left(\beta_{\mathrm{p}}=1\right) \\
\mathrm{v}_{\mathrm{o}}=20 \mathrm{v}_{\mathrm{A}} \quad\left(\mathrm{V}_{\mathrm{A}} / \mathrm{c}=1 . \times 10^{-4}\right)
\end{array}
$$

$$
\begin{gathered}
-\cdot \text { Cold }_{\mathrm{b}}=\mathrm{T}_{\mathrm{p}} \\
\beta_{\mathrm{p}}=0.35-0.80
\end{gathered}
$$

Fig. 9. Observed transport ratios and polarization comparison with the values derived from linear kinetic theory for the cold beam right-hand resonant instability $\left(k c / \omega_{p}=0.05\right)$. The parameters used to define various cold beam distributions are given above. The first set of parameters describe the "typically observed" distribution, while the remaining events correspond to changes in density, velocity and temperature of the beam. Solid dots correspond to events $7-10$ in Table 3 (right-handed waves in plasma rest frame) 
and that mode identification based only on magnetic polarization quantities does not give a complete picture of the waves' characteristics and can lead to mode identification of waves whose polarization may agree with theoretical predictions even when other properties can diverge from those of the theoretical modes. This accentuates the importance of using transport ratios in addition to the polarization to characterize and identify the observed fluctuations.

That the observed wave properties are close to the values given by the theory shows that, as a first approach, the kinetic model is a good approximation to describe a system such as the foreshock and that the large amplitude fluctuations observed there keep some similarities with the small-fluctuations described in the theory. However, this theory does not describe the compressive nature of the observed waves, neither does it consider the effects that wave-particle coupling, may have in modifying linear mode properties. A more accurate description of the waves requires a theory in which nonlinear processes such as the steepening that the waves suffer while approaching the shock are taken into account. Thus, the ideal way to perform wave identification would be using quasi-linear theory or kinetic (or hybrid) simulations to evaluate wave identifiers.

The presence of diffuse ion distributions in all the observed intervals where the waves are identified as Alvénic is consistent with previous observations (Hoppe and Russell, 1983) and with the fact that hot beam distributions are necessary to generate the left-handed modes (Gary, 1985, 1991, 1993). The origin of large amplitude compressive ULF waves observed in the foreshock has been a major subject of discussion. Originally, due to the curved geometry of the bow shock, it was suggested that the waves were the result of the interaction of cold ion beams reflected from the quasi-perpendicular portion of the shock, growing to the large observed amplitudes as they propagate downstream in the foreshock (Greenstadt et al., 1977). Numerical works have studied the nonlinear steepening of the waves generated by this mechanism (Winske and Leroy, 1984; Akimoto et al., 1991; Onsager et al., 1991). However, a cold beam can not generate waves that are left-handed in the plasma frame and the presence of such fluctuations can not be explained in this scenario. Further work (Burgess, 1989; Thomas et al., 1990; Winske et al., 1990) showed that the quasi-parallel portion of the bow shock is intrinsically turbulent, presenting the necessary background to develop a scheme in which the waves are produced locally in the quasi-parallel shock. In this scenario (Scholer, 1993; Dubouloz and Scholer, 1995), reflected ions from the quasi-parallel bow shock generate right-hand polarized perturbations in the foreshock able to scatter the original beam to a more diffuse distribution, that can then supply the free energy for the generation of lefthanded modes. Therefore, the observed left-handed modes are generated by the hot beam instability while it is possible to have right-handed magnetosonic modes generated either by reflected or by diffuse distributions.
Reflected beams are not observed all the time in the foreshock, and as they eventually are scattered to a hot beam distribution, it seems most likely that the majority of the right-handed waves observed deep in the foreshock are also generated by the hot resonant instability. An alternative description of wave generation and steepening is based on the nonlinear evolution of ring-beam instabilities in the foreshock (Killen et al., 1995; Fuselier, 1995).

By comparing the observed transport ratios with the properties derived for the two hot beam instabilities we consider that the waves are generated locally by the interaction between the diffuse ions with the solar wind, in a scheme such as the one described already (Dubouloz and Scholer, 1995). However, as mentioned before, some of the right-handed waves may be generated by reflected beams or ring-beams in other regions of the foreshock and be swept by the solar wind to regions where diffuse ions are observed.

Finally, we believe that a comparison with a theory including large fluctuations (i.e. simulations) could be more satisfactory to describe the nature of waves observed in the Earth's foreshock. Part of future work would be to make a similar analysis using simultaneous data from different spacecraft to have a more complete description of the waves in the foreshock, and study the nonplanarity noted here.

Acknowledgements. We thank Andrew Coates of MSSL for providing the AMPTE-UKS ion data as well as Malcolm Dunlop of Imperial College of Science, Technology and Medicine who provided the AMPTE-UKS magnetometer data. Thanks to $\mathrm{K}$. Rönmark, for allowing the use of WHAMP. We are also grateful to David Burgess, Jim Leckband, Filippo Pantellini and Andrew Fazakerley for useful discussions. X.B.C. is grateful for the generous support of the National University of México and for the hospitality of the Astronomy Unit at QMW college while finishing this manuscript.

Topical Editor K.-H. Glaßimeier thanks D.Krauss-Varban and D.S. Orlowski for their help in evaluating this paper.

\section{Appendix: definitions}

\section{Transport ratios}

Using linear Vlasov theory we evaluate the polarization, the parallel compressibility, the Alfvén ratio, the crosshelicity, and two magnetic ratios $C_{B}$ and $R_{n c}$ for the right-hand and left-hand resonant ion beam instabilities that can be generated in the foreshock by the interaction of the hot diffuse ions with the solar wind.

The magnetic polarization with respect to $\mathbf{k}$ is

$P=i \frac{\delta B_{s}}{\delta B_{A}}$

where $\delta$ indicates the amplitudes of fluctuating quantities that vary in time as $\exp (-i \omega t)$. The directions A and $\mathbf{S}$ correspond to the typical directions of fluctuation for Alfvén waves and magnetosonic waves respectively. $\mathbf{A}$ is perpendicular to the plane defined by $\mathbf{k}$ and the unperturbed magnetic field $\mathbf{B}_{\mathbf{0}}$ and $\mathbf{S}$ is in the plane $\mathbf{k}-\mathbf{B}_{\mathbf{0}}$, 
perpendicular to k. $P$ measures how magnetosonic $(|P|>1)$ or how Alfvénic $(|P|<1)$ the waves are. For circularly polarized waves $|P|$ is equal to unity. Re $\mathrm{P}>$ $0(<0)$ corresponds to waves that are right-hand (lefthand) polarized, with $\delta \mathbf{B}$ rotating around $\mathbf{B}_{\mathbf{o}}$ in the same sense as electrons (protons). In all the figures, $P_{B_{o} k}=|P|(-|P|)$ when the wave is right-handed (lefthanded). This polarization can be related to the magnetic compression $C_{B B}$ (Krauss-Varban et al., 1994) and gives similar information to the generalized ellipticity (Orlowski et al., 1994a) and the polarization in Stix (1992).

We evaluate two magnetic ratios (Denton et al., 1995) the first one measures the magnetic compression of the wave,

$C_{B}=\frac{<\delta B_{\|} \delta B_{\|}>}{<\delta \mathbf{B} \cdot \delta \mathbf{B}>}$

where $\delta B_{\|}$is the component of the fluctuating magnetic field along $\mathbf{B}_{\mathbf{0}}$. As in Gary and Winske (1992), all quantities $\langle a b\rangle$ indicate the correlation between $a$ and $b$ for a given pair of $\omega$ and $\mathbf{k}$.

To measure the component of the fluctuating field out of the plane $\mathbf{k}-\mathbf{B}_{\mathbf{o}}$ with respect to the total magnetic fluctuation, we evaluate the noncoplanar ratio (similar to the "transverse ratio" of Song et al., 1994)

$R_{n c}=\frac{<\delta B_{A} \delta B_{A}>}{<\delta \mathbf{B} \cdot \delta \mathbf{B}>}$

The Alfvén ratio (Matthaeus and Goldstein, 1982) is

$r_{A j}=\frac{\left\langle\delta \mathbf{v}_{j} \cdot \delta \mathbf{v}_{j}>\right.}{\langle\delta \mathbf{b} \cdot \delta \mathbf{b}>}$

where $\delta \mathbf{b}=\left(\mathbf{B}-\mathbf{B}_{\mathbf{o}}\right) /\left(4 \pi m_{p}\left(n_{p}+n_{b}\right)\right)^{1 / 2}$ and the subscript $j$ denotes the $\mathrm{j}$-species in the plasma $(p, e$, and $b$ refer to the proton core, the electrons and the ion beam). For an Alfvén wave $\delta \mathbf{v}_{p} / \mathbf{v}_{A}= \pm \delta \mathbf{B} / \mathbf{B}_{\mathbf{0}}$ so that $r_{A_{p}}=1$. Thus $r_{A_{p}}$ is a measure of the "Alfvén-ness" of lowfrequency waves. However, the Alfvén ratio is also near unity for a fast mode wave propagating in a low beta plasma, so $r_{A_{p}}$ cannot uniquely identify Alfvén waves.

The cross-helicity is defined as

$\sigma_{c j}=\frac{2<\delta \mathbf{v}_{j} \cdot \delta \mathbf{b}>}{<\delta \mathbf{v}_{j}^{2}>+<\delta \mathbf{b}^{2}>}$

$\sigma_{c p}=-1(1)$ for an Alfvén mode propagating parallel (anti-parallel) to the unperturbed magnetic field. In the case of the fast magnetosonic mode, $\left|\sigma_{c p}\right| \sim 1$ for propagation at small angles to $\mathbf{B}_{\mathbf{0}}$, presenting the same change in sign as the Alfvén mode for parallel and antiparallel propagation. For this mode $\left|\sigma_{c p}\right|$ tends to zero as the angle of propagation increases (Gary, 1993). $\sigma_{c j}$ can be very useful to determine uniquely the direction of propagation of low frequency waves in the foreshock. The angle between $\mathbf{k}$ and $\mathbf{B}_{\mathbf{0}}$ determined by the minimum variance method (Hoppe et al., 1981) presents a $180^{\circ}$ ambiguity. This can be resolved using the sign of the cross-helicity to establish whether the waves are propagating in the direction of the magnetic field (at relatively small angles) or in the opposite direction (assuming they lie on the magnetosonic or Alfvén branch).

The "parallel compressibility" (Lacombe et al., 1992), is defined as

$C_{\| j}=\frac{<\delta n_{j} \delta B_{\|}>}{n_{j} B_{o}} \frac{B_{o}^{2}}{<\delta B_{\|} \delta B_{\|}>}$

The name "parallel compressibility" can sometimes be misleading, as in the case of the Alfvén mode at small angles of propagation when the values of $C_{\| p}$ can be above unity. This occurs due to a small value of $\delta B^{2}$, so that the value of $C_{\| p}$ is reflecting the noncompressibility of the mode rather than its compressibility. There are other transport ratios associated with the compressibility of the waves, such as the "compressibility" (Gary, 1986) the "compressional ratio" (Song et al., 1994) and the "parallel phase ratio" (Denton et al., 1995). The compressibility and the compressional ratio may provide a more general estimation of how compressive a mode is, as their evaluation does not involve $\delta B_{\|}$. However, at small angles of propagation, the compressibility of the fast and Alfvénic modes is very similar and small and is not a useful identifier. The combined evaluation of $C_{\| p}$ and $C_{B}$ provides a fair estimation of how compressive a mode can be.

\section{Magnetic field analysis}

Here we define the magnetic quantities used in addition to the transport ratios to characterize different modes. The normalized amplitude of the magnetic fluctuations is $\delta B_{n}=[|\delta \mathbf{B}|] /\left|\mathbf{B}_{\mathbf{o}}\right|$, where $\delta \mathbf{B}=\mathbf{B}-\mathbf{B}_{\mathbf{0}}$ and subscript "o" is used to indicate the non-perturbed values of $\mathbf{B}, \mathbf{v}$, and $n$ (taken as the average value over the entire analysis interval). Quantities between[] indicate averages over the sliding window of width $T_{a}$.

We use the minimum variance technique (Hoppe et al., 1981) to determine the direction of propagation of the waves. This direction is well determined (apart from a $180^{\circ}$ ambiguity) if the waves are well constrained to the plane of polarization. When this happens, the minimum variance eigenvalue, $\lambda_{3}$ is much less than the intermediate one, $\lambda_{2}$, i.e. $e \equiv \lambda_{3} / \lambda_{2} \ll 1$. Thus an analysis based on a plane wave description may be misleading when $e \sim 1$. We evaluate $\theta_{k B o}$ and $\theta_{i B o}$, the angles between $\mathbf{k}$ and $\mathbf{B}_{\mathbf{0}}$ and between $\mathbf{i}$, the direction of maximum variation, and $\mathbf{B}_{\mathbf{0}}$.

The ellipticity is defined as $\epsilon=\sqrt{\lambda_{1} / \lambda_{2}}$ (Wilkinson et al., 1993), where $\lambda_{1}$ is the maximum variance eigenvalue. For a circularly polarized wave $\epsilon=1$.

We evaluate the magnitude of the magnetic polarization with respect to $\mathbf{k}$ (see transport ratios definitions), in terms of $\sigma_{B_{S}}$, and $\sigma_{B_{A}}$, the variances along $\mathbf{S}$ and $\mathbf{A}$ as $\left|P_{B_{o} k}\right|=\sigma B_{s} / \sigma B_{A}$.

To estimate the predominant sense of polarization of the waves we follow the method of Elaoufir et al. (1990). Considering the instantaneous rotation of $\delta \mathbf{B}_{\mathbf{i}}=\mathbf{B}_{\mathbf{i}}-\mathbf{B}_{\mathbf{0}}$, with $\mathbf{B}_{\mathbf{i}}$ the $\mathrm{i}^{-}{ }^{\text {th }}$ magnetic field vector, it is possible to define an instantaneous axis of rotation $\mathbf{K}_{\mathbf{i}}=\delta \mathbf{B}_{\mathbf{i}} /\left|\delta \mathbf{B}_{\mathbf{i}}\right| \times \delta \mathbf{B}_{\mathbf{i}+\mathbf{1}} /\left|\delta \mathbf{B}_{\mathbf{i}+\mathbf{1}}\right|$. The amount of rotation 
between consecutive measurements is $\theta_{i}=\sin ^{-1}\left|\mathbf{K}_{\mathbf{i}}\right|$. The rotation is positive when $\mathbf{B}_{\mathbf{0}} \cdot \mathbf{K}_{\mathbf{i}}>0$ and negative when this product is negative. Positive (negative) rotations correspond to right (left) hand polarization with respect to $\mathbf{B}_{\mathbf{0}}$ in the spacecraft frame. We evaluate the predominant (mean) polarization rate $P_{\text {mean }}=\left[\theta_{i} / t_{i}\right]$, where $t_{i}$ is the time between consecutive measurements. When large rotations occur, the mean polarization does not precisely indicate the sense of rotation for the majority of the vectors, thus we also evaluated the sense of the rotation, $s r$, given by number, i.e., the direction that corresponds to the majority of the rotations. The average "rotational frequency" within each sliding window, is defined as $f_{r}=\left[\left|\theta_{i}\right| / 2 \pi t_{i}\right]$ which provides a crude estimate of the wave frequency. This estimate tends to reflect the highest frequency signal rather than that of the largest amplitude.

\section{References}

Akimoto, K., D. Winske, T. G. Onsager, M. F. Thomsen, and S. P. Gary, Steepening of parallel propagating hydromagnetic waves into magnetic pulsations, J. Geophys. Res., 96, 17599 17607, 1991.

Blanco-Cano, X., Waves and particles upstream of the Earth's bow shock, PhD Thesis, University of London, London, 1995.

Burgess, D., Cyclical behavior at quasi-parallel collisionlesss shocks, Geophys. Res. Lett., 16, 345-349, 1989.

Coates, A. J., J. A. Bowles, R. A. Gowen, B. K. Hancock, A. D. Johnstone, and S. J. Kellock, AMPTE-UKS 3-D ion experiment, IEEE Trans. Geosci. Remote Sensing, GE-23(3), 287292, 1985

Denton, R.E., S. P. Gary, X. Li, B. J. Anderson, J. W. LaBelle, and M. Lessard, Low-frequency fluctuations in the magnetosheath near the magnetopause, J. Geophys. Res., 100, 5655-5679, 1995.

Dubouloz, N., and M. Scholer, Two-Dimentional simulations of magnetic pulsations upstream of the Earth's bow shock, J. Geophys. Res., 100, 9461-9474, 1995.

Elaoufir, J., A. Mangeney, T. Passot, C. C. Harvey, and C. T. Russel, Large amplitude MHD waves in the Earth's proton foreshock, Ann. Geophysicae, 8, 297-314, 1990.

Ellison, D. C., Shock acceleration of diffuse ions at the Earth's bow shock: acceleration efficiency and $\mathrm{A} / \mathrm{Z}$ enhancement, J. Geophys. Res., 90, 29-38, 1985.

Fairfield, D. H., Bow shock associated waves observed in the far upstream interplanetary medium, J. Geophys, Res.,, 74, 3541, 1969.

Fuselier, S.A., Ion distributions in the Earth's foreshock upstream from the bow shock, Adv. Spac. Res., 15, 43-52, 1995.

Gary, S. P., Electromagnetic ion beam instabilities: hot beams at interplanetary shocks, Astrophys. J., 288, 342-352, 1985.

Gary, S. P., Low frequency waves in a high-beta collisionless plasma:polarization, compressibilityy and helicity, J. Plasma Phys., 35, 431-447, 1986.

Gary, S. P., Electromagnetic ion/ion instabilities and their consequences in space plasmas: a review, Space Sci. Rev., 56, 373-415, 1991.

Gary, S.P., Theory of Space Plasma Microinstabilities, Cambridge University Press, New York, 1993.

Gary, S. P., and D. Winske, Correlation function ratios and the identification of space plasma instabilities, J. Geophys. Res., 97, 3103-3111, 1992

Giacalone, J., D. Burgess, S. J. Schwartz and D. C. Ellison, Ion injection and acceleration at parallel shocks: comparisons of self-consistent plasma simulations with existing theories, Astrophys. J., 402, 550-559, 1993.
Greenstadt, E. W., Oblique, parallel, and quasi-parallel morphology of collisionless shocks, in Collisionless Shocks in the Heliosphere: Reviews of Current Research, Geophys. Monogr. Ser., vol 35, Eds B. T. Tsurutani and R. G. Stone, pp. 169-184, AGU, Washington, D. C., 1985.

Greenstadt, E. W., I. M. Green, G. T. Inoye, A. J. Hundhausen, S. J. Bame, and I. B. Strong, Correlated magnetic field and plasma observations of the Earth's bow shock, J. Geophys. Res., 73, 5160, 1968.

Greenstadt, E. W., C. T. Russell, V. Formisano, P. C. Hedgecock, F. L. Scarf, M. Neugebauer, and R. E. Holzer, Structure of a quasi-parallel, quasi-laminar bow shock, J. Geophys. Res., 82, 651-666, 1977.

Hoppe, M. M., and C. T. Russel, Plasma rest frame frequencies and polarizations of the low frequency upstream waves: ISEE 1 and 2 observations, J. Geophys. Res., 88, 2021-2028, 1983.

Hoppe, M. M., C. T. Russell, L. Frank, T. Eastman, and E. Greenstadt, Upstream hydromagnetic waves and their association with backstreaming ion population: ISEE 1 and 2 observations, J. Geophys. Res, 86, 4471-4492, 1981.

Killen, K., N. Omidi, D. Krauss-Varban, and H. Karimabadi, Linear and nonlinear properties of ULF waves driven by ring-beam distribution functions, J. Geophys. Res., 100, 5835-5852, 1995.

Krauss-Varban, D., N. Omidi, and K. B. Quest, Mode properties of low frequency waves: kinetic theory versus Hall-MHD, J. Geophys. Res., 99, 5987-6009, 1994.

LaBelle, J., R. A. Treuman, and E. Marsch, Elsässer variable analysis of fluctuations in the ion foreshock and undisturbed solar wind, J. Geophys. Res., 99, 65-71, 1994.

Lacombe, C. E. Kinzelin, C. C. Harvey, D. Hubert, A. Mangeney, J. Elaoufir, D. Burgess, and C. T. Russell, Nature of the turbulence observed by ISEE 1-2 during a quasi-perpendicular crossing of the Earth's bow shock, Ann. Geophysicae, 8, 489502,1990

Lacombe, C., F. G. E. Pantellini, D. Hubert, C. C. Harvey, A. Mangeney, G. Belmont, and C. T. Russel, Mirror and Alfvénic waves observed by ISEE 1-2 during crossings of the Earth's bow shock, Ann. Geophysicae, 10, 1-13, 1992.

Le, G., Generation of upstream ULF waves in the Earth's foreshock, PhD Thesis, University of Calif., Los Angeles, 1991.

Matthaeus, W. H., and M. L. Goldstein, Measurement of the rugged invariants of magnetohydrodynamic turbulence in the solar wind, J. Geophys. Res., 87, 6011-6028, 1982.

Muret, P., and N. Omidi, Analysis of steepened magnetosonic waves using wavelet transforms and neural networks, J. Geophys. Res., 100, 23465-23479, 1995.

Onsager, T. G., D. Winske, and M. F. Thomsen, Interaction of a finite-length ion beam with a background plasma: reflected ions at the quasi-parllel bow shock, J. Geophys Res., 96, 1775-1788, 1991.

Orlowski, D. S., C. T. Russell, D. Krauss-Varban, and N. Omidi, Growing "Alfvenic" modes in the upstream region of Saturn, J. Geophys. Res., 99, 19-25, 1994a.

Orlowski, D. S., C. T. Russell, D. Krauss-Varban, and N. Omidi, A test of the Hall-MHD model: application to low-frequency upstream waves at Venus, J. Geophys. Res., 99, 169-178, 1994b.

Paschmann, G., N. Sckopke, I. Papamastorakis, J. R. Asbridge, S. J. Bame, and J. T. Gosling, Characteristics of reflected and diffuse ions upstream from the earth's bow shock, J. Geophys. Res., 86, 4355-4364, 1981.

Rönmark, K., WHAMP-waves in homogeneous, anisotrophic multicomponent plasmas, Kiruna Geophys, Inst. Kiruna, Sweden, Rep. 179, 1982.

Russell, C. T., J. G. Luhmann, R. C. Elphic, D. J. Southwood, M. F. Smith and A. D. Johnstone, Upstream waves simultaneously observed by ISEE and UKS, J. Geophys. Res., 92, 7354-7362, 1987.

Scholer, M., Upstream waves, schoklets, SLAMS and the cyclic behavior of oblique quasi-parallel collisionless shocks. J. Geophys. Res., 98, 47-57, 1993. 
Schwartz, S. J., and D. Burgess, Quasi-parallel shocks: a patchwork of three dimensional structures, Geophys. Res. Lett., 18, $373-$ 376, 1991.

Schwartz, S. J., D. Burgess, and J. J. Moses, Low frequency waves in the Earth's magnetosheath, Ann. Geophysicae, 14, 1134-1150, 1996.

Song, P., C. T. Russell, and S. P. Gary, Identification of lowfrequency fluctuation in the terrestrial magnetosheath, $J$. Geophy. Res., 99, 6011-6025, 1994.

Southwood, D. J., W. A. C. Mier-Jedrezejowicz, and C. T. Russell, The fluxgate magnetometer for the AMPTE UK subsatellite, ISEE Trans. Geosci. Remote Sens., GE-23, 301-304, 1985.

Stix, T. H., Waves in Plasmas, American Institute of Physics, New York, 1992.

Thomas, V. A., D. Winske, and N. Omidi, Re-forming supercritical quasi-parallel shocks: 1 . One- and two-dimensional simulations, J. Geophys. Res., 95, 18,809-18,819, 1990.

Thomsen, M. F., Upstream suprathermal ions, in Collisionless shocks in the Heliosphere: Reviews of Current Research, Geophys. Monogr. Ser., vol 35, eds. B. T. Tsurutani and R. G. Stone, pp. 253-270, AGU, Washington, D.C., 1985.
Thomsen, M. F., J. T. Gosling, S. J. Bame, and C. T. Russell, Magnetic pulsations and the quasi-parallel shock, J.Geophys. Res., 95, 957-966, 1990.

Tsurutani, B.T., and P. Rodriguez, Upstream waves and particles: an overview on ISEE results, J. Geophys. Res., 86, 4319-4324, 1981.

Tsurutani, B. T., R. M. Thorne, E. J. Smith, J. T. Gosling, and H. Matsumoto, Steepened magnetosonic waves at comet Giacobini-Zinner, J. Geophys. Res., 92, 11,074-11,082, 1987.

Wilkinson, W. P., A. K. Pardaens, S. J. Schwartz, D. Burgess, H. Lühr, R. L. Kessel, M. Dunlop, and C. J. Farrugia, Nonthermal ions and associated magnetic field behaviour at a quasiparallel Earth's bow shock, J. Geophys. Res., 98, 3889-3905, 1993.

Winske, D., and M. M. Leroy, Diffuse ions produced by electromagnetic ion-beam instabilities, J. Geophys. Res., 89, 2673-2688, 1984.

Winske, D., V. A. Thomas, and N. Omidi, Re-forming supercritical quasi-parallel shocks: 2. Mechanism for wave generation and front re-formation, J. Geophys. Res., 95, 18,82118,832, 1990. 